\title{
Interaction between lean, fat and bone masses among patients with chronic kidney disease; a cross-sectional comparative study
}

\author{
Silva EH ${ }^{1}$, Wickramatilake CM${ }^{2}$, Lekamwasam S ${ }^{3}$, Mudduwa LKB ${ }^{4}$, Ubayasiri RA ${ }^{5}$ \\ ${ }^{1}$ Department of Medical Laboratory Science, Faculty of Allied Health Sciences, University of Ruhuna, Galle, Sri Lanka. \\ ${ }^{2}$ Department of Biochemistry, ${ }^{3}$ Department of Medicine, ${ }^{4}$ Department of Pathology, Faculty of Medicine, University of \\ Ruhuna, Galle, Sri Lanka. \\ ${ }^{5}$ Department of Vascular and Transplant Surgery, Teaching Hospital Karapitiya, Galle, Sri Lanka.
}

Correspondence: Ms. Eranga H. Silva

e-mail:ehsilva@ahs.ruh.ac.lk

https://orcid.org/0000-0003-0139-8869

Submitted on 29.07.2021 and accepted for publication on 06.08.2021

\begin{abstract}
Introduction: Interactions between three major body compartments; lean, fat and bone masses are essential for the maintenance of optimum bodily functions. This interaction is disturbed by factors such as advancing age, disease and drugs. The aim of the study was to understand the interaction of body compartments in chronic kidney disease (CKD) to optimise disease outcome.
\end{abstract}

Methods: Fifty patients (38 males) with CKD awaiting kidney transplant at Teaching Hospitals, Karapitiya and Kandy and 50 age and gender matched control subjects were compared. Socio-demographic data, social habits and anthropometric measurements with blood pressure was obtained. Both patients and controls underwent DEXA scan to assess body composition.

Results: Mean(SD) age of the patients was 48(9.6) years. In both groups; patients with CKD and participants in the control group, total body lean mass (TBLM) showed significant correlations with total body bone mineral density (TBBMD) (patients: $\mathrm{r}=0.38, p=0.008$ ) (controls: $\mathrm{r}=0.57, p=<0.001$ ) and total body bone mineral content (TBBMC) (patients: $\mathrm{r}=0.62, p=<0.001$ ) (controls: $\mathrm{r}=0.78, p=<0.001$ ). No significant correlations were observed between total body fat mass (TBFM) and TBBMD or TBBMC. When regression models were fitted with TBBMD and TBBMC as dependent variables and TBLM as the independent variable, $1 \mathrm{~kg}$ difference in TBLM was associated with $0.007 \mathrm{~g} / \mathrm{cm}^{2}(p=0.02)$ and $0.010 \mathrm{~g} / \mathrm{cm}^{2}(p=0.012)$ change in TBBMD in patients and controls, respectively. Furthermore, $1 \mathrm{~kg}$ difference in TBLM was associated with $32 \mathrm{~g}(p=0.02)$ and $33 \mathrm{~g}$ $(p=0.018)$ difference in TBBMC in patients with CKD and participants in the control group, respectively.

Conclusions: Similar to participants in the control group, TBLM is a better predictor of TBBMC and TBBMD compared to TBFM and TRFM in patients with end stage renal disease (ESRD) and this knowledge can be used in health promotion programs to improve bone health of these patients.

Key words: Body composition, bone mineral content, bone mineral density, end stage renal disease, fat mass, lean mass

\section{Introduction}

Chronic kidney disease (CKD) is a global health problem with an overall global prevalence of $13.4 \%$ (1). Derangement of bone mineral metabolism occurs in all stages of CKD, it can cause physical immobility, musculoskeletal pain and bone fractures, resulting in increased mortality, morbidity and poor quality of life (2). This group of disorders initially named as renal osteodystrophy (ROD) is now renamed as CKD - mineral and bone disorder (CKD-MBD) by considering the extra-skeletal manifestations associated with the condition (3). 
The reduction of bone mineral density (BMD) which is a major determinant of bone strength (2) starts in the early stages of CKD and continues with the progressive decrease of renal functions and patients with end stage renal disease (ESRD) have a higher risk of fracture (2).

The associations between bone mass, lean and fat masses, are complex and vary according to age, gender, hormonal status, disease stage and drug usage. These associations are more complex in patients with CKD and there are uncertainties about the influence of obesity on bone mass among patients with CKD (4). We believe that understanding the interactions between three main body compartments; lean, fat and bone masses among patients with CKD is relevant in their long-term management. Such information would assist in designing nutritional interventions and life style modifications in patients with CKD. However, studies carried out to assess the association between bone, fat and lean masses among patients with ESRD on routine haemodialysis are sparse and we were unable to find previous studies conducted among Sri Lankans.

\section{Methods}

Patients with ESRD (stage V) awaiting renal transplant at Teaching Hospitals, Karapitiya and Kandy were included in the study. Consecutive patients were included after obtaining informed written consent. Patients who had previous fracture, metal implants or renal transplant were excluded from the study. Approval for the study protocol was obtained from the Ethics Review Committee of Faculty of Medicine, University of Ruhuna, Galle, Sri Lanka. Fifty (38 males) age and gender matched controls were recruited for the study from the neighbourhood of the patients (neighbourhood controls) after excluding renal impairment by serum creatinine estimation. In selecting controls, chronic illnesses or following special life style modification programmes such as intense dietary interventions were excluded.

An interviewer administered questionnaire was used to collect data and additional information was gathered from personal medical records. The duration of the disease was calculated from the time of the first detection to the time of the interview. The duration of the dialysis was calculated from the first dialysis to the last dialysis session. The socio-demographic data collected included age, monthly income, education status, smoking and alcohol consumption. Patients were categorised as non-smoker (never smoked), past smoker or current smoker depending on the smoking status. Same type of categorisation was used to describe the alcohol consumption.

Standard protocols were followed when obtaining anthropometric measurements. A portable stadiometer was used to measure the height to the nearest $1 \mathrm{~cm}$. Weight was measured to the nearest $0.1 \mathrm{~kg}$ using a beam balance. A non-stretchable tape was used to measure waist and hip circumferences to the nearest $1 \mathrm{~cm}$ and waist to hip ratio was calculated.

Systolic and diastolic blood pressures were measured using a sphygmomanometer (Matsuoka Japan) after allowing the study subjects to rest for 15 minutes, an average of the two consecutive measurements was taken for analysis.

All patients underwent body composition analysis with dual energy x-ray absorptiometry (DEXA) (Hologic Discovery QDR, USA) scan. Bone related measurements included total body BMD (TBBMD), total body bone mineral content (TBBMC), BMDs in femoral neck, total hip and lumbar spine (L1 to L4, anterior-posterior projection). Total body lean mass (TBLM), total body fat mass (TBFM) and truncal fat mass (TRFM) were also measured using the analytical software (Version 12.4) provided by Hologic.

SPSS statistical software was used for the statistical analysis. Descriptive data were presented as mean (SD) while categorical data as frequencies and percentages. Non-normally distributed data were presented as median and interquartile range. Independent sample t-test was used to compare the anthropometric variables and body composition variables between the patients and controls. The association between body compartments were tested using Pearson correlation. Probability of $<0.05$ was considered as statistically significant.

\section{Results}

Fifty patients (38 males) with CKD and fifty age and 
sex matched individuals (controls) were recruited for the study. The median (interquartile range) durations of disease and dialysis (those were on HD) were 24 (12 - 36) and 8 (4 - 12) months, respectively.

The majority of patients had completed school education up to the G.C.E. Ordinary Level and most of them had a monthly income of less than LKR 25,000. A considerable number of patients $(26 \%)$ had no regular income.

There were no current smokers or current alcohol consumers among patients, however, there were 23
(46\%) past smokers and $26(52 \%)$ past consumers of alcohol (Table 1).

Collectively, a greater proportion of controls (54\%) had completed G.C.E. Advanced Level or university education. The majority of controls (64\%) had monthly income between LKR 25,000 to 60,000. Among controls, 26\% were current smokers while $72 \%$ were current alcohol consumers (Table 1).

Table 1: Data on Socio-demographic characteristics and social habits

\begin{tabular}{lcccc}
\hline & $\begin{array}{c}\text { Patients with } \\
\text { CKD } \\
\mathbf{n}=\mathbf{5 0}(\%)\end{array}$ & $\begin{array}{c}\text { Participants in the } \\
\text { Control group } \\
\mathbf{n}=\mathbf{5 0}\end{array}$ \\
\hline Education
\end{tabular}


Participants in the control group and patients with CKD were similar with regards to height, weight and waist circumference. Compared to participants in the control group, patients with CKD had lower BMI and hip circumference and higher waist to hip ratio and systolic and diastolic blood pressures. Although patients with CKD had lower TBFM and TBLM and higher TRFM and body fat percentage compared to participants in the control group, only the difference in TBLM was significant. Furthermore, TBBMD, TBBMC and regional BMDs at the hip region were significantly lower among patients with CKD compared to the participants in the control group (Table 2).
In both participants in the control group and patients with CKD, TBBMD, TBBMC and regional BMDs showed significant positive correlations with TBLM. Compared to CKD patients, the correlation seen between these measurements were greater among participants in the control group (Table 3). Furthermore, inverse correlations were seen between percentage fat mass and TBBMC and TBBMD measurements. The inverse correlations seen between TBFM and TBBMC/ TBBMD values were not significant.

Table 2: Comparison of anthropometry, blood pressure and body composition between patients with CKD and participants in the control group

\begin{tabular}{|c|c|c|c|c|c|}
\hline \multirow{2}{*}{$\begin{array}{l}\text { Measurement } \\
\text { Age (completed years) }\end{array}$} & \multicolumn{2}{|c|}{$\begin{array}{l}\text { Patients with CKD } \\
\qquad \begin{array}{c}(\mathrm{n}=\mathbf{5 0}) \\
\text { Mean (SD) }\end{array}\end{array}$} & \multicolumn{2}{|c|}{$\begin{array}{l}\text { Participants in the } \\
\text { Control group } \\
(\mathbf{n}=\mathbf{5 0}) \\
\text { Mean (SD) }\end{array}$} & \multirow{2}{*}{$\begin{array}{r}\text { p value } \\
0.72\end{array}$} \\
\hline & 45 & $(10)$ & 44 & $(10)$ & \\
\hline Height (m) & 1.62 & $(0.09)$ & 1.61 & $(0.08)$ & 0.54 \\
\hline Weight (kg) & 58.4 & $(11.9)$ & 62.3 & $(11.1)$ & 0.09 \\
\hline $\operatorname{BMI}\left(\mathrm{kg} / \mathrm{m}^{2}\right)$ & 22.1 & $(3.7)$ & 23.9 & $(3.7)$ & 0.01 \\
\hline $\mathrm{WC}(\mathrm{cm})$ & 82.7 & $(10.5)$ & 82.2 & $(9.00)$ & 0.79 \\
\hline $\mathrm{HC}(\mathrm{cm})$ & 89.7 & $(8.3)$ & 93.8 & $(7.6)$ & 0.01 \\
\hline WHR & 0.92 & $(0.06)$ & 0.87 & $(0.06)$ & $<0.001$ \\
\hline SBP (mmHg) & 163.7 & $(27.4)$ & 119.8 & $(13.9)$ & $<0.001$ \\
\hline $\mathrm{DBP}(\mathrm{mmHg})$ & 97.4 & $(14.7)$ & 79.6 & $(11.9)$ & $<0.001$ \\
\hline eGFR & 9.4 & $(3.5)$ & 94.7 & $(21.4)$ & $<0.001$ \\
\hline TBFM (g) & 16113.1 & $(5229.9)$ & 16622.8 & $(5242.9)$ & 0.63 \\
\hline TRFM (g) & 8144.3 & $(3162.3)$ & 7548.8 & $(2617.9)$ & 0.31 \\
\hline Body fat percentage $(\%)$ & 27.8 & $(6.2)$ & 27.0 & $(7.0)$ & 0.54 \\
\hline TBBMC (g) & 1925.8 & $(370.5)$ & 2067.3 & $(336.5)$ & 0.05 \\
\hline TBLM (g) & 39011.2 & $(7879.4)$ & 42444.1 & $(8051.7)$ & 0.034 \\
\hline $\operatorname{TBBMD}\left(\mathrm{g} / \mathrm{cm}^{2}\right)$ & 1.06 & $(0.11)$ & 1.11 & $(0.09)$ & 0.02 \\
\hline $\operatorname{SBMD}\left(\mathrm{g} / \mathrm{cm}^{2}\right)$ & 0.93 & $(0.23)$ & 0.94 & $(0.15)$ & 0.88 \\
\hline FNBMD $\left(\mathrm{g} / \mathrm{cm}^{2}\right)$ & 0.72 & $(0.14)$ & 0.85 & $(0.13)$ & 0.001 \\
\hline $\operatorname{THBMD}\left(\mathrm{g} / \mathrm{cm}^{2}\right)$ & 0.86 & $(0.13)$ & 1.02 & $(0.14)$ & $<0.001$ \\
\hline
\end{tabular}

Abbreviations: BMI - body mass index; WC - waist circumference; HC - hip circumference; WHR - waist hip ratio; SBP systolic blood pressure; DBP - diastolic blood pressure; eGFR - estimated glomerular filtration rate; TBFM - total body fat mass; TRFM - truncal fat mass; BMC - bone mineral content; TBLM - total body lean mass; TBBMD - total body bone mineral density; SBMD - spine bone mineral density; FNBMD - femoral neck bone mineral density; THBMD - total hip bone mineral density. 
Table 3: Correlations between measures of body composition among cases and controls

\begin{tabular}{lllllll}
\hline & & TBBMC & TBBMD & SBMD & FNBMD & THBMD \\
\hline TBFM & Cases & 0.076 & -0.093 & -0.057 & 0.088 & -0.123 \\
& Controls & 0.155 & 0.157 & $0.326^{*}$ & 0.157 & 0.275 \\
\hline \multirow{2}{*}{ TBLM } & Cases & $0.616^{* *}$ & $0.377^{* *}$ & 0.399 & $0.421^{*}$ & 0.217 \\
& Controls & $0.788^{* *}$ & $0.570^{* *}$ & $0.490^{* *}$ & $0.547^{* *}$ & $0.613^{* *}$ \\
\hline
\end{tabular}

** Correlation is significant at the 0.01 level. * Correlation is significant at the 0.05 level.

Abbreviations: TBFM, to tal body fat mass; TBLM, total body lean mass; TBBMC, total body bone mineral content; TBBMD, total body bone mineral density; SBMD, spinal bone mineral density; FNBMD, femoral neck bone mineral density; THBMD, total hip bone mineral density.

When a regression model was fitted with TBBMD and TBBMC as dependent variables and TBLM as the independent variable, $1 \mathrm{~kg}$ difference is TBLM was associated with $0.007 \mathrm{~g} / \mathrm{cm}^{2}(p=0.02)$ and $0.010 \mathrm{~g} / \mathrm{cm}^{2}(p=0.012)$ change in TBBMD in patients and controls, respectively. Furthermore, $1 \mathrm{~kg}$ difference in TBLM was associated with $32 \mathrm{~g}$ $(p=0.02)$ and $33 \mathrm{~g}(p=0.018)$ different in TBBMC in patients and controls, respectively.

\section{Discussion}

This study showed significant positive relationships between TBLM, and total body and regional BMDs among both participants in the control group and patients with CKD. The correlations seen among participants in the control group were greater when compared with those among CKD patients. These findings indicate that muscle mass has greater influence than fat mass on total body and regional BMDs in this age group but the association is lesser among patients with CKD.

Information related to body composition analyses among patients with CKD is sparse. The observations made among CKD patients in this study are consistent with the limited literature previously published on patients with CKD on dialysis $(4,5)$. Previous studies have reported that lean mass is a better predictor of bone quality of patients on haemodialysis and healthy individuals $(4,6)$. The study by Negri, et al., using sixty patients on peritoneal dialysis (45 women and 20 men), found lean body mass to be the only body composition measurement to show a positive correlation with BMC among males and postmenopausal females (6).

The link between lean mass and BMD / BMC is plausible and possibly a result of dynamic load resulting from muscle contractions on bone formation $(7,8)$. Further, the distortions and tensions caused by muscle mediated mechanical forces on bone tissue act on osteocytes to upsurge bone strength (9). In addition, muscle mass produces stretching of collagen fibers and periosteum at the interface, resulting in the stimulation of bone growth, and higher blood flow to bone lead to an increase in bone strength, as blood flows to limbs at a level proportional to muscle mass (10).

Although it was thought that increased body fat, through peripheral synthesis of oestrogen, enhances BMD (11), recent studies have shown fat mass to have negative effects on bone mass (12). The concept of beneficial effects of mechanical loading of increased body weight over bone mass is challenged by these studies (13). In addition, it is evident that increased visceral fat is more harmful for bone tissue (12). A study by Fournie et al, conducted among patients on peritoneal dialysis revealed that surrogate markers of visceral fat such as waist circumference, truncal fat and leg fat were inversely related to cortical volumetric BMD (13). We found percentage fat mass to be inversely and significantly associated with BMD while inverse associations between TBFM and BMD did not reach statistical significance, probably due to sample size limitation and these findings are concordant with previous studies (13). Further, 
the specific role of truncal fat over the total body fat in determining BMD cannot be ruled out.

Kirchengast and Huber, found that association between soft tissue body compartments and bone varies according to the gender (14). Their findings showed that fat mass is the best predictor of bone mass among women whereas lean mass is the best predictor among men (14). In our study, the majority of patients were men $(76 \%)$ and the results obtained may have been affected by that.

One of the major strengths of our study was that we compared the associations between fat and lean masses with bone measurements in patients with $\mathrm{CKD}$, age and gender matched participants in the control group. Another is the use of DEXA scan to measure the body composition parameters. It had allowed the measurement of both body composition and bone parameters non-invasively with a minimum radiation and high accuracy. Small sample size with less female representation and cross-sectional study design were the major limitations of the study.

\section{Conclusions}

Similar to participants in the control group, TBLM is a better predictor of TBBMC and TBBMD compared to TBFM and TRFM in patients with ESRD and this knowledge can be used in health promotion programs to improve bone health of these patients.

Data used for this publication will be shared upon request. Authors have no conflict of interest to declare.

\section{Acknowledgment}

The study has been funded by University Grants Commission block grant for strengthening research (2016) awarded by University Grants Commission, Sri Lanka. (GrantNo; RU/PG/R/16-06)

\section{References}

1. Hill NR, Fatoba ST, Oke JL, et al. Global prevalence of chronic kidney disease - a systematic review and metaanalysis. PloS one. 2016; 11(7): e0158765.
2. Aggarwal HK, Jain D, Yadav S, Kaverappa V. Bone mineral density in patients with predialysis chronic kidney disease. Renal Failure. 2013; 35(8): 1105-1111.

3. Moe S, Drüeke T, Cunningham J, Goodman W, et al. Definition, evaluation, and classification of renal osteodystrophy: a position statement from Kidney Disease: Improving Global Outcomes (KDIGO). Kidney International. 2006; 69(11): 1945-53.

4. Marinho SM, Wahrlich V, Mafra D. Association between body composition and bone mineral density in men on hemodialysis. Am JMed Sci. 2015; 350(4): 286-289.

5. Negri AL, Barone R, Bogado CE, Zanchetta JR. Relationship between weight, body composition, and bone mass in peritoneal dialysis. Nefrologia. 2005; 25(3): 269-74.

6. Pelletier S, Bacchetta J, Boutroy S, et al. The relation between adipokines, body composition, and bone health measured with hr-pqct in haemodialysis patients. Nephrology Dialysis Transplanation. 2015; 30(suppl 3): iii598.

7. Lanyon LE, Rubin CT. Static vs dynamic loads as an influence on bone remodelling. J Biomech. 1984; 17(12): 897-905.

8. Forwood MR, Turner CH. Skeletal adaptations to mechanical usage: results from tibial loading studies in rats. Bone. 1995; 17(4 Suppl):197-205S.

9. Frost HM. Bone's mechanostat: a 2003 update. Anat Rec A Discov Mol Cell Evol Biol. 2003; 275(2):1081-1101.

10. Kaji H. Interaction between Muscle and Bone. J Bone Metab. 2014; 21(1): 29-40.

11. Bogl LH, Latvala A, Kaprio J, et al. An investigation into the relationship between soft tissue body composition and bone mineral density in a young adult twin sample. JBone Miner Res. 2011;26(1): 79-87.

12. Liu YH, Xu Y, Wen Y-B, et al. Association of weightadjusted body fat and fat distribution with bone mineral density in middle-aged Chinese adults: a cross-sectional study. PLOS One. 2013; 8(5): e63339.

13. Fournie C, Pelletier S, Bacchetta J, et al. The relationship between body composition and bone quality measured with HR-pQCT in peritoneal dialysis patients. Peritoneal Dialysis International. 2017; 37(5): 548-555.

14. Kirchengast S, Huber J. Sex-specific associations between soft tissue body composition and bone mineral density among older adults. Annals of Human Biology. 2012;39(3): 206-13. 